\title{
The influence of pitch and birefringence on nematicon propagation at the disclination lines in chiral nematic liquid crystals
}

\author{
Michał Kwaśny, Bartłomiej W. Klus and Urszula A. Laudyn \\ ${ }^{I}$ Faculty of Physics, Warsaw University of Technology, Koszykowa 75, 00-662 Warszawa,
}

Received December 18, 2014; accepted December 30, 2024; published December 31, 2014

\begin{abstract}
In this work we study the influence of pitch and birefringence on nematicon propagation at the disclination lines in a wedge shaped planarly oriented sample. We demonstrate that changing the structure parameters we can efficiently bend soliton trajectory, as a result of reflection.
\end{abstract}

The concept of optical solitons is inherently associated with many nonlinearity-driven propagational effects. Spatial optical solitons, i.e. nondispersive and nondiffractive beams propagating in nonlinear media, are formed thanks to an interplay between diffraction, which tends to spread the beam, and the self-induced nonlinear refractive index change of the medium, which focuses the beam [1]. Among others, spatial optical solitons in nematic liquid crystals (NLCs), commonly referred to as nematicons, play a crucial role [2]-[3]. The main source of interest includes high nonlinearity stemming from molecular reorientation. This type of nonlinearity is derived from changes in the refractive index in a liquid crystalline medium caused by the intensity of light. With an increase in light intensity the birefringence axis of NLC molecules changes its direction and a gradient profile of the refraction index is formed. Nematicons have been demonstrated and investigated in several NLCs geometries, including planar [4], homeotropic [5], twisted and chiral [6-9]. They can be used to create reconfigurable optical circuits created by light alone, where all-optical switching or processing is achieved through the evolution and interaction of many soliton beams [10-12]. Light guiding, coupling, routing and combining are essential to optical signal processing, and this crucial light manipulation leads to important development of optical communication systems. By increasing the complexity of crystalline geometry, more advanced light controls could be achieved for wide applications. Among them, there has been growing interest in research on the use of chiral nematic liquid crystals (chiral nematics, cholesterics, ChNLCs) with a helical arrangement of molecules [8-9]. A very promising configuration is obtained using a wedge-shaped slit and a chiral substance. The latter leads to the formation of the Grandjean steps, parallel bands separated by disclination lines [13-14]. It has already been demonstrated [8-9, 1516] that non-uniform distribution of the refractive index along the thickness of the liquid crystal in a wedge cell enables to easily control the direction of signal propagation. In so prepared a cell, disclination lines are formed, i.e. the lines where distribution of the refractive index is discontinuous. This work provides an insight into nematicon propagation in a highly disturbed area around the disclination line in mixtures with different pitch and birefringence values, respectively. The main idea of the presented experimental results is related to reflection, refraction and change in the direction of nematicon propagation in ChNLCs in a wedge cell. In this work we investigated low-birefringent NLC 1110 [17] with $n_{\mathrm{o}}=1.452$ and $n_{\mathrm{e}}=1.498$ at room temperature and at $\lambda=1.064 \mu \mathrm{m}$ with a chiral dopant at a weight concentration of about $5 \%$ and $2 \%$, which yields a pitch of about $8 \mu \mathrm{m}$ and $20 \mu \mathrm{m}$, respectively and a typical birefringent NLC E7 (Merck) with $n_{\mathrm{o}}=1.504$ and $n_{\mathrm{e}}=1.695$ at room temperature and $\lambda=1.064 \mu \mathrm{m}$ with a chiral dopant with a pitch of about $20 \mu \mathrm{m}$
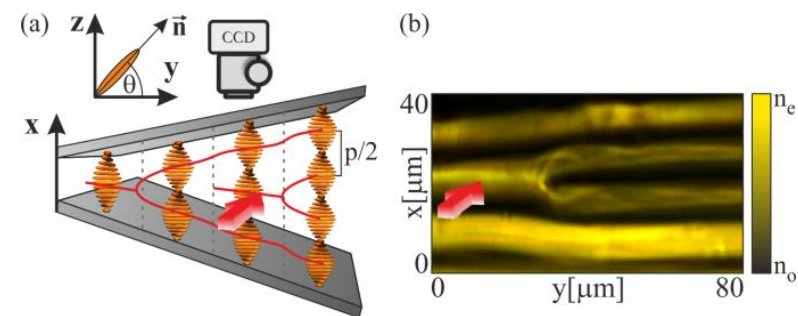

Fig. 1. (a) Sketch of an experimental setup and chiral structure confined in a wedge shaped cell with a refractive index profile for an incident light beam polarized along the y-axis propagating in z-direction; (b) photo taken in the $y x$-plane (while illuminated by the white light) showing refractive index distribution close to the disclination line.

The experimental setup and cell geometry are sketched in Fig. 1a. The chiral structure is sandwiched between two glass plates making a small angle and treated for planar anchoring in the same direction. As a consequence, the thickness of a sample is gradually changed along the sample length. Nematicons were generated by a monochromatic beam from an Nd:YAG laser operating with the wavelength $\lambda=1.064 \mu \mathrm{m}$ focused on the NLC layer by a $20 \times$ micro-objective into a laser spot of several micrometers in the sample $\left(\mathrm{w}_{0} \sim 3.8 \mu \mathrm{m}\right)$. The input linear polarization of the beam was controlled by a halfwavelength plate. The light intensity distribution of a propagating beam inside the cell was acquired by collecting the scattered light out of the top slide by a CCD 
camera. In the investigated configuration, a light beam propagates in the $z$-direction, parallel to the glass plates. A nematicon is generated by injecting a polarized beam with polarization parallel to the $y$-axis with a wave vector parallel to the $z$-axis. According to Fig. 1a, when a $y$ polarized light beam is launched perpendicular to the helical axis, it experiences a refractive index continuously varying across the $x$-direction, taking the range from ordinary to extraordinary value. As a consequence, in geometry considered, one can distinguish high index regions throughout the cell thickness, where the light beam (of proper polarization) tends to propagate [7]. The number of such regions is determined by the pitch value and is equal to $2 d / p$ (where $\mathrm{d}$ is the cell thickness), which means that the true periodicity is the half-pitch. The resultant texture depends on a helix pitch $p$ and the NLC birefringence. The pitch $p$ determines the thickness and number of layers and the birefringence determines the contrast between the layers. It means that the medium is stratified along the $\mathrm{x}$ direction, and if the ChNLCs is confined in a wedge cell, the refractive index distribution is affected by a disclination line along $x$. Disorder along the $x$-axis is the difference in the refractive index for the same value of $x$, which results in an index change for $y$ polarized beam propagating across the disclination line. The refractive index perceived by the impinging beam from the right side of disclination is different than sensed by the beam from the left side of the disclination line (for the same $x$ value). As a consequence, the nematicon created at the right side of disclination collapses after passing it [8].

In an ideal situation, namely ideal refractive index distribution (Fig. 1b), the beam propagating on the right side of the disclination line sees an extraordinary refractive index, while on the left side, for the same $x$ value the beam sees an ordinary refractive index. It means that when the beam strikes the disclination line from right to left, it experiences a refractive index shift and the beam undergoes reflection and is repelled by the interface. In low birefringent chiral nematic liquid crystals mixtures, the minimal optical power required for self-trapping is higher than in NLCs with a typical value of $\Delta n$ [18-19] and also due to the Frank elastic constant $\mathrm{K}_{22}$,which is higher in 1110 ChNLC than E7.

Figure 2 compares the influence of pitch and birefringence in the case of nematicon striking the disclination line. Firstly, we compare low birefringent ChNLC $(\Delta n=0.04)$ with two different pitch values ( 8 and $20 \mu \mathrm{m}$ respectively). Launching a beam at the same angle to the disclination line, we observe that in the case of a smaller pitch the beam is split into two, one part is reflected from the disclination line and the second one propagates through disclination without any changes in its direction. The low pitch means a thinner optical layer in comparison to the beam waist. Increasing the pitch value causes the whole beam to be reflected for the same angle of incidence.

(a)
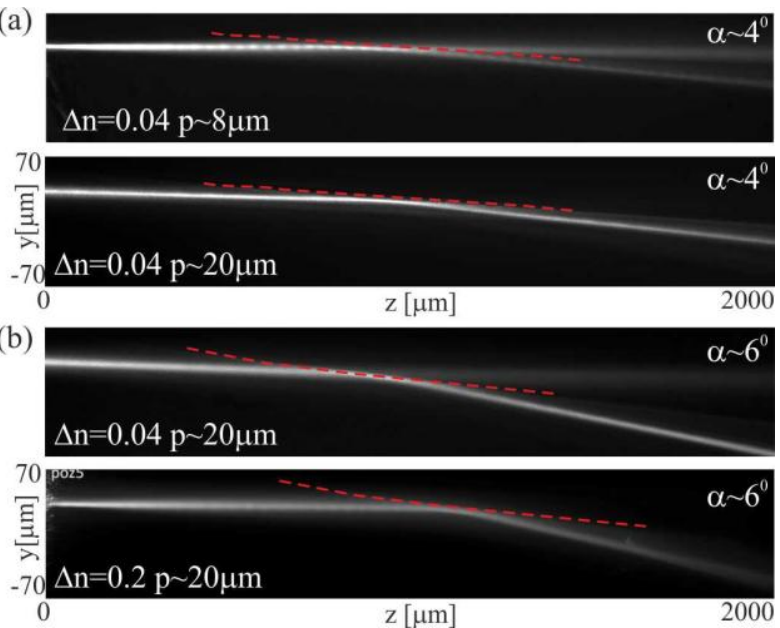

Fig. 2. Experimental results taken for mixtures with different pitch and birefringence values, showing the beam reflectance at the disclination line (a) low-birefringent 1110 NLC with pitch 8 and $20 \mu \mathrm{m}$ respectively; (b) comparison between low and typical birefringent NLCs with an equal pitch $(\sim 20 \mu \mathrm{m})$

Moreover, keeping the pitch value constant and increasing the birefringence, we observe similar behaviour. In this case the pitch was $20 \mu \mathrm{m}$, and the beam was injected at an angle of $6^{\circ}$ to the disclination line. The angle was chosen so that in the case of small birefringence, the beam is divided into two: reflected and passing beam. Increasing the birefringence causes the entire beam to be reflected due to a higher reflectance index. The results depicted in Fig. 2 can be summarized by the fact that a thicker layer (higher pitch value) and a higher effective refractive index inside the layer means stronger light beam guiding, i.e. the mode is more strongly guided in the layer. In addition to the above, we have also analysed the stability with increasing input beam power. In the case of low birefringent and small pitch (1110 ChNLC with $8 \mu \mathrm{m}$ pitch), increasing the input beam power leads to energy coupling between the divided beams (Fig. 3a). Higher power means stronger reorientation of molecules in both sides of the disclination line and consequently lower difference in refractive index distribution. As a consequence, the reflected part of the beam is less confined and a blurred diffractive background is observed. A further increase in beam power leads to the self-focusing of a passing beam and the whole beam passes the disclination line without changing its propagation direction.

In the case of $1110 \mathrm{ChNLC}$ with a higher pitch (wider layer) as well as E7 ChNLC increasing beam power has little effect on the stability of the reflected beam or its direction of propagation. Increasing the input beam also leads to stronger reorientation. However, since the whole beam is reflected, it actually increases the difference in 
refractive index distribution on both sides of the disclination line.
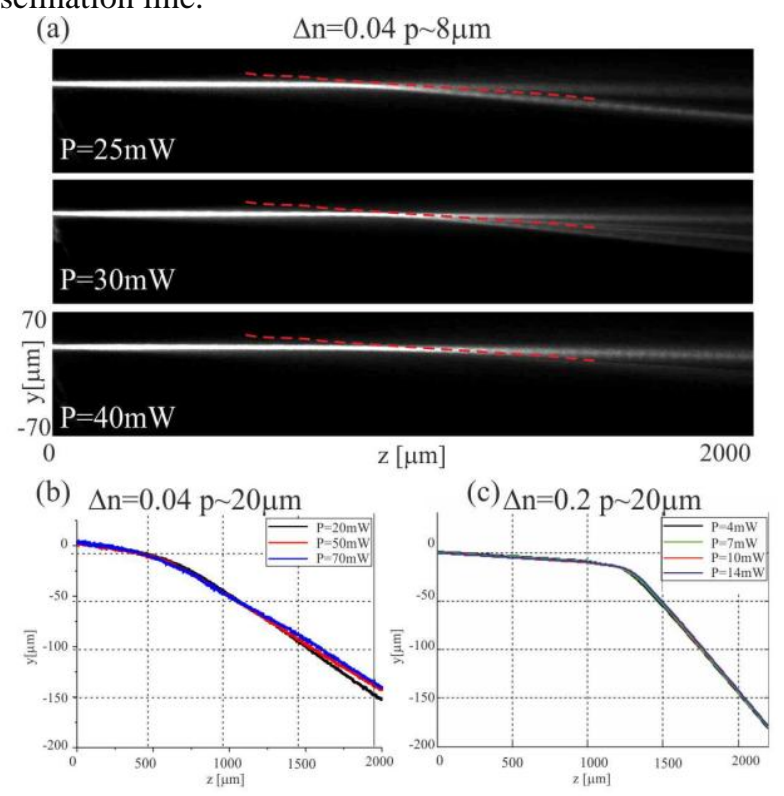

Fig. 3. The stability of obtained results from Fig. 2 with increasing incident beam power (a) photos of light beam propagation and nematicon trajectory in mixture 1110 with pitch $8 \mu \mathrm{m}$; (b)-(c) stability of mixtures with equal pitch $20 \mu \mathrm{m}$ but different birefringence: low birefringent 1110 mixture (b) and typical birefringent E7 (c).

Furthermore, depending on the beam launching angle with respect to the disclination lines, it is possible to control the direction of nematicon propagation. Figure 4 presents the changes in the nematicon trajectory as a function of angle between the incident beam and the disclination line. Changes in the angle between the beam and the disclination line were obtained by turning the ChNLC cell with a proper angle without changing incident beam geometry. The maximal angle of incidence depends on a refractive index shift on both sides of disclination, thus depends directly on the birefringence of used NLC mixtures. Using the principal of refraction and reflection, the maximum angle in respect to the disclination line for which the beam undergoes reflection estimated to be about $10^{\circ}$ in low-birefringent 1110 ChNLCs and about $20^{\circ}$ in E7. This assumption, however, does not include absorption, scattering loses or beam walk-off, which highly affects the maximal angle. Indeed, in the experiment, the reflection of a beam from the disclination line was observed for angles up to $8^{\circ}$ in 1110 and up to $12^{\circ}$ in E7 ChNLC.
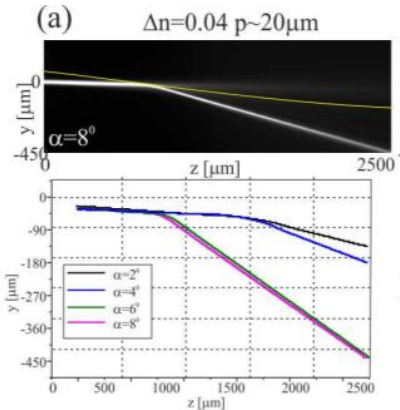

Fig. 4. Comparison between low (a) and typical birefringent (b) NLC with equal pitch and different angle of incident beam in respect to disclination line.

To conclude, we have studied the influence of pitch and birefringence on nematicon propagation at the disclination lines in a wedge shaped planarly oriented sample. We have demonstrated that changing structure parameters can be successfully employed to bend the soliton trajectory as a result of reflection.

This work was supported by the National Science Centre under the decision number DEC- 2012/05/D/ST7/00147.

\section{References}

[1] S. Trillo, W.Torruellas, Spatial Solitons (Berlin, Spriger Verlang 2001).

[2] G. Assanto, M.A. Karpierz, Liq. Cryst. 36, 1161 (2009).

[3] G. Assanto, Nematicons, (John Willey\& Sons 2012).

[4] M. Peccianti, G. Assanto, A. De Luca, C. Umeton, I. C. Khoo, Appl. Phys. Lett. 77, 7 (2000).

[5] M.A. Karpierz, M. Sierakowski, M. Swillo, T. Wolinski, Mol. Cryst. Liq. Cryst. 320, 157 (1998).

[6] U.A. Laudyn, M. Kwasny, M.A. Karpierz, Appl. Phys. Lett. 94, 091110 (2009).

[7] U.A. Laudyn, P.S. Jung, K.B. Zegadło, M.A. Karpierz and G. Assanto, Opt. Letters 39, 6399 (2014).

[8] U.A. Laudyn, M.A. Karpierz Appl. Phys. Lett.103, 221104 (2013.)

[9] M. Kwaśny, U. Laudyn, K. Rutkowska, M. Karpierz J. of Nonl. Opt. Phys. Mat. 23, 1450042 (2014).

[10] M. Peccianti, C. Conti, G. Assanto, A. De Luca, C. Umeton, Appl. Phys. Lett. 81, 3335 (2002).

[11] S. Serak, N.V. Tabiryan, M. Peccianti, G. Assanto, IEEE Photon. Technol. Lett. 18 (12), 1287 (2006).

[12] A. Fratalocchi, A. Piccardi, M. Peccianti, G. Assanto, Opt. Letters 32, 1447 (2007).

[13] H.S. Kitzerrow, Ch. Bar, Chirality in Liquid Crystals (New York, Springer 2001).

[14] P. Oswald, P. Pieranski, Nematic and Cholesteric Liquid Crystals: Concepts and Physical Properties Illustrated by Experiments (Taylor \& Francis 2005).

[15] M. Peccianti, A. Dyadyusha, M. Kaczmarek, G. Assanto, Nature Photonics 2, 737 (2006).

[16] M. Peccianti, G. Assanto, A. Dyayusha, M. Kaczmarek, Opt. Letters 32, 271 (2007).

[17] 1110 nematic mixture was synthesized by Prof. R. Dabrowski at Military University of Technology in Warsaw

[18] M. Kwasny, U.A. Laudyn, F. A. Sala, A. Alberucci, M.A. Karpierz and G. Assanto, Phys. Rev. A 86, 013824 (2012).

[19] B. Klus, U.A. Laudyn, M.A. Karpierz, B. Sahraoui, Opt. Express 22, 30257 (2014). 